\title{
Performance characteristics of magnetic resonance cholangiography in the staging of malignant hilar strictures
}

\author{
S H Zidi, F Prat, O Le Guen, Y Rondeau, G Pelletier
}

\begin{abstract}
Background-Magnetic resonance cholangiography (MRC) is currently under investigation for non-invasive biliary tract imaging.

Aim-To compare MRC with endoscopic retrograde cholangiography (ERC) for pretreatment evaluation of malignant hilar obstruction.

Methods-Twenty patients (11 men, nine women; median age 74 years) referred for endoscopic palliation of a hilar obstruction were included. The cause of the hilar obstruction was a cholangiocarcinoma in 15 patients and a hilar compression in five (one hepatocarcinoma, one metastatic breast cancer, one metastatic leiomyoblastoma, two metastatic colon cancers). MRC (T2 turbo spin echo sequences; Siemens Magnetomvision $1.5 \mathrm{~T}$ ) was performed within 12 hours before ERC, which is considered to be the ideal imaging technique. Tumour location, extension, and type according to Bismuth's classification were determined by the radiologist and endoscopist.
\end{abstract}

Results-MRC was of diagnostic quality in all but two patients $(90 \%)$. At ERC, four patients $(20 \%)$ had type I, seven $(35 \%)$ had type II, seven $(35 \%)$ had type III, and two $(\mathbf{1 0 \% )}$ had type IV strictures. MRC correctly classified $14 / 18(78 \%)$ patients and underestimated tumour extension in four $(22 \%)$. Successful endoscopic biliary drainage was achieved in 11/17 attempted stentings $(65 \%)$, one of which was a combined procedure (endoscopic + percutaneous). One patient had a percutaneous external drain, one had a surgical bypass, and in a third a curative resection was attempted. Effective drainage was not achieved in six patients $(30 \%)$. If management options had been based only on MRC, treatment choices would have been modified in a more appropriate way in $5 / 18(28 \%)$ patients with satisfactory MRC.

Conclusion-MRC should be considered for planning treatment of malignant hilar strictures. Accurate depiction of high grade strictures for which endoscopic drainage is not the option of choice can preclude unnecessary invasive imaging. (Gut 2000;46:103-106)

Keywords: hilar tumours; endoscopic retrograde cholangiopancreatography; magnetic resonance cholangiography; stenting
Malignant hilar obstructions are caused by tumours involving the major confluence of the hepatic ducts and the upper third of the common bile duct. Obstruction may result from metastasis to hilum confines, but more often arises primarily from the extrahepatic bile duct. Primary bile duct carcinoma represents $2 \%$ of all cancers found at autopsy and $10-20 \%$ of all hepatic malignancies. ${ }^{1-3}$ Primary hilar carcinomas account for more than $40 \%$ of cholangiocarcinomas. ${ }^{1}$

Hilar tumours are usually classified according to the invasion of primary and secondary confluences. ${ }^{4}$ Tumours metastatic to the porta hepatis are by definition non-resectable for cure and consequently require palliative therapy. ${ }^{5}$ This is also true for most primary lesions because of regional or distant malignant spread, patient infirmity, or preference at the time of diagnosis. ${ }^{6}{ }^{7}$ Endoscopic or percutaneous biliary drainage may be preferred to palliative surgery in non-resectable tumours, resulting in lower postoperative mortality and morbidity, ${ }^{6}$ but many advanced cases are beyond any treatment possibility. When tumour spread is restricted to the major confluence of the hepatic ducts, endoscopic (or percutaneous) stenting can be successfully performed resulting in effective biliary drainage. ${ }^{89}$ However, drainage failure after contrast injection often occurs in high grade strictures and may result in severe sepsis and worsen a patient's condition. ${ }^{10}$ There is a lack of an accurate non-invasive method for depicting bile duct anatomy which could improve considerably the management of these patients by suggesting the most appropriate treatment choice before any contrast is injected into the bile ducts.

Magnetic resonance cholangiography (MRC) is a developing non-invasive technique for biliary tract imaging. Several reports have shown its ability to display the biliary tree by combining the advantages of projectional and cross sectional views, ${ }^{11}$ and many reports have shown a high accuracy in detection of intrahepatic biliary ductal dilatation ${ }^{12}{ }^{13}$ and in suggesting the site and cause of obstruction. ${ }^{14}{ }^{15}$

We investigated whether MRC could be relied upon as the sole determinant of hilar stricture type and subsequently improve patient management by precluding direct cholangiography in those with presumably unsuccessful endoscopic

Abbreviations used in this paper: MRC, magnetic resonance cholangiography; ERC, endoscopic retrograde cholangiography; CT, computed tomography. 
drainage manoeuvres. We performed a prospective blinded comparison of MRC and endoscopic retrograde cholangiography (ERC) in patients referred to our centre for endoscopic stenting.

\section{Patients and methods \\ PATIENTS}

Twenty patients were included in the study. The inclusion criteria were: (a) clinical suspicion of hilar malignancy on the basis of clinical, biochemical, and imaging data (transcutaneous ultrasonography and computed tomography (CT) scan); (b) obstructive jaundiced patients referred for endoscopic biliary stenting; (c) visible hilar mass and/or dilated intrahepatic bile ducts with a non-dilated common duct at CT scan or ultrasonography. Non-jaundiced patients and non-dilated intrahepatic ducts were excluded.

There were 11 men and nine women, mean (SD) age 69.5 (12) years (range 42-100, median 74 years). All patients were jaundiced. The other clinical features were a weight loss of more than $10 \%$ during the three months preceding inclusion (11 patients, 55\%), pruritus (eight patients, $40 \%$ ), fever (five patients, $25 \%$ ), abdominal pain (five patients, $25 \%$ ), and ascites (four patients, 20\%). Mean (SD) bilirubinaemia at the time of inclusion was 312 (125) $\mu \mathrm{mol} / 1$. Intrahepatic biliary dilatation was constantly present on ultrasonography or CT scan. Dilatation involved both sides of the liver symmetrically in 14 cases, and was asymmetric in six cases. One patient had severe cholangitis and septic shock at presentation.

MRC

All patients were scheduled for MRC 12 hours before ERC, after informed consent was obtained. They did not receive antiperistaltic agents but they were fasting in order to reduce motion artefacts from bowel peristalsis. MRC was performed on a 1.5 unit Siemens Magnetomvision using a body coil (the main standard machine coil). The magnetic resonance cholangiograms were acquired using nonbreath-hold, fat suppressed, respiratory triggered turbo spin echo sequences. Two dimensional turbo spin echo imaging was performed in the axial and coronal planes. Imaging parameters were: repetition time/echo time, 4500 milliseconds/138 milliseconds; matrix, $174 \times 256$; acquisition time, one minute 53 seconds for each pile of sections; section thickness, $5 \mathrm{~mm}$ with no overlap. Only MRC sequences were used. We did not use magnetic resonance angiography or tissue sequences. Tumour mass was assessed from CT and ultrasonography scans. The radiologists performing MRC were blinded to previous imaging findings; the only information they had was that of obstructive jaundice symptoms.

YARDSTICK IMAGING

ERC was performed under general anaesthesia using TJF 100 or TJF 130 duodenoscopes (Olympus, Tokyo, Japan). All the patients received intravenous antibiotic prophylaxis with amoxicillin and clavulanic acid. Cholangio-
Table 1 Agreement between endoscopic retrograde cholangiography (ERC) and magnetic resonance cholangiography (MRC) on stricture type

\section{Stricture}

type No of correct $M R C$ gradings/ERC grading

\begin{tabular}{ll}
\hline I & $4 / 4$ \\
II & $6 / 7$ (1: undergraded as type I) \\
III & $4 / 7$ (2: undergraded as type II; 1: MRC \\
& $\begin{array}{l}\text { unsuccessful }) \\
\text { IV }\end{array}$ \\
& $\begin{array}{l}\text { unsuccessful }) \\
\text { unsaded as type III; } 1: \text { MRC }\end{array}$ \\
\end{tabular}

Overall agreement: $70 \%$ (78\% after exclusion of two technically unsuccessful MRC attempts).

grams allowed stricture grading according to Bismuth's classification. The endoscopists were blinded to the MRC results. When appropriate, stenting was performed after sphincterotomy, using one or two 11.5 or $10 \mathrm{~F}$ endoprostheses.

\section{IMAGE ANALYSIS}

Magnetic resonance cholangiograms and direct cholangiograms were separately interpreted by radiologists and endoscopists in a blinded fashion. Both teams evaluated the following items: intrahepatic bile duct dilatation (left, right, or both sides), common bile duct dilatation, and stricture grading according to Bismuth's classification (I, an obstruction at the upper third of the common bile duct but still patent communication between the right and left lobes; II, an obstruction of the major confluence and suppression of biliary communication between the right and left lobes; III, more extensive infiltration into the liver with segmentally occluded ducts on one side of the liver; IV, extensive and bilateral tumour spread). ${ }^{4}$ In addition, the cause of the obstruction or the presence of a tumour mass (intrahepatic or at the hilus confines) was also noted by the radiologists.

\section{Results}

The causes of malignant obstruction were: histologically proved cholangiocarcinoma in nine patients (45\%); highly probable cholangiocarcinoma in six $(30 \%)$ (high grade dysplasia at cytological brushing); malignant compression to hilar confines in five (25\%) (one hepatocarcinoma, one metastatic breast cancer, one metastatic leiomyoblastoma, two metastatic colon cancers).

Direct cholangiography allowed stricture type classification in all patients. Four patients (20\%) had type I, seven patients $(35 \%)$ had type II, seven patients (35\%) had type III, and two patients $(10 \%)$ had type IV strictures.

Table 2 Management and results of palliative drainage attempts

\begin{tabular}{lll}
\hline Stricture type & Management & Results \\
\hline I & 2: surgery & $4:$ success \\
& 2: endoscopic stenting & \\
II & 5: endoscopic stenting & 5: success \\
& 1: combined procedure & $1:$ success \\
III & 1: percutaneous drainage & 1: success \\
& 7: endoscopy & $4:$ success \\
IV & 2: endoscopy & 3: failure \\
\end{tabular}

* "Rendez-vous" technique combining percutaneous insertion of a guidewire and endoscopic insertion of a stent. 

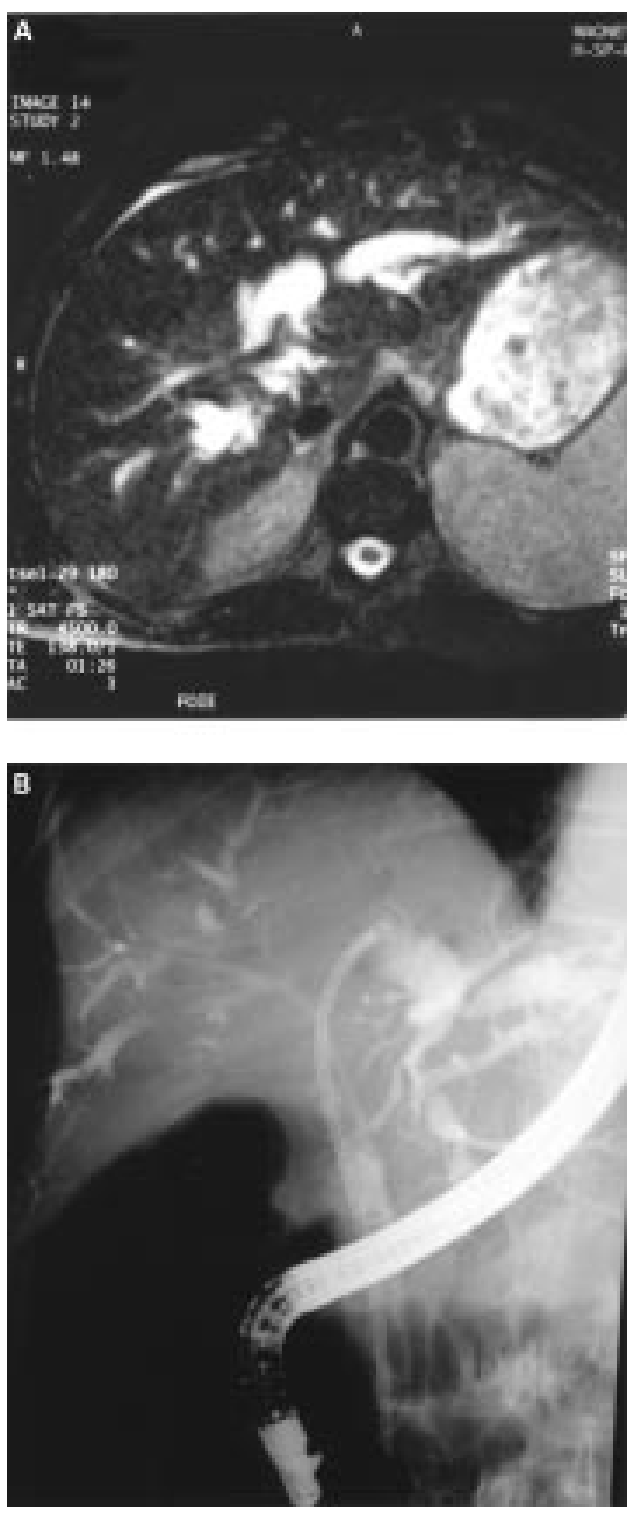

Magnetic resonance cholangiograms were not of diagnostic quality in two patients, with ascites causing severe image blurring. Intrahepatic bile duct dilatation was present at MRC in $17 / 18$ patients, and common bile duct dilatation was present in $3 / 18$. In $14 / 18(78 \%)$ patients, MRC stricture staging was in accordance with that of direct cholangiography (fig 1). In four patients (22\%), MRC underestimated the stricture type (table 1). Overall agreement between ERC and MRC was $70 \%$ if the two technically unsuccessful MRC examinations are included. ERC/MRC agreement was $91 \%$ for type I and II strictures (10/11 patients).

Table 2 shows the management of the patients. Two patients bearing a type I stricture underwent surgery with complete resolution of jaundice. One patient with a type II stricture was managed with percutaneous external drainage only. Thus endoscopic biliary drainage, although considered for all the patients, was actually attempted in $17 / 20(85 \%)$. Of these 17 patients, four $(23 \%)$ had advanced disease at ERC (two grade III and two grade IV strictures) and retrograde stenting was considered unworkable. These patients were subsequently managed medically and received broad spectrum antibiotics (ceftazidime) to prevent cholangitis after biliary opacification. Endoscopic stenting was attempted in the 13 other patients (two type I, six type II, and five type III). Successful guidewire access through the malignant stenosis and stricture balloon dilatation were performed in 12 patients, with subsequent endoscopic stent insertion. One patient had a combined procedure (endoscopic and percutaneous drainage). Satisfactory biliary drainage was obtained in $11 / 17$ cases $(65 \%)$, with a $50 \%$ or more decrease in serum bilirubin, and in two patients, only partial jaundice resolution (less than 50\% decrease in bilirubinaemia) was obtained after stenting. In patients with grade I and II strictures, the success rate of endoscopic intubation was $8 / 8(100 \%)$, as against $4 / 9(44 \%)$ in patients with grade III and IV strictures (table 2). Patient survival time in our series ranged from three days to 18 months; median survival was three months.

\section{Discussion}

Malignant hilar tumours are usually unresectable at the time of diagnosis. ${ }^{6} 7$ Although resection offers the only chance of cure, the five year survival rate after potentially curative resection for hilar cholangiocarcinoma has been reported to be from 0 to $22 \%,{ }^{216}$ and the resectability rate from 20 to $30 \%{ }^{6}{ }^{16}$; there is no indication for surgery in patients with hilar metastases. Hence palliative non-surgical treatment of obstructive jaundice is often the main treatment for most patients at the time of diagnosis, as radical excision of hilar tumours is precluded by the patient's general condition, tumour extension into both lobes of the liver, portal vein, or hepatic artery tumour encasement, or distant metastasis.

When communication persists between the right and left hepatic ducts (Bismuth's type I), the endoscopic insertion of a single stent is admittedly the best way to ensure drainage, although some type I stricture patients may be candidates for surgical resection or bypass. When the tumour extends to the origin of the left and right ducts, curative resectability is uncommon, and selective intubation of both sides is still generally possible by the endoscopic route, ${ }^{8}$ although transcutaneous stenting is also a valuable option. ${ }^{9}$ In type III tumours, the transcutaneous approach is often preferred to endoscopy for its ability to intubate more than two sectors selectively, while type IV is generally considered beyond therapeutic resource. Endoscopically placed stents are associated with fewer complications than transhepatic stenting. ${ }^{17}{ }^{18}$ However, successful endoscopic drainage is rare in type III strictures, and procedure related complications (mainly cholangitis and subsequent renal failure) are common. ${ }^{10}$ Filling of undrained segments with contrast during ERC is problematic, as aggressive wire and catheter manipulation if a drainage attempt is considered increases the risk of subsequent cholangitis, and the effectiveness of antibiotics on 
Table 3 Consequences of magnetic resonance cholangiography (MRC) on patient management

\begin{tabular}{llll}
\hline $\begin{array}{l}\text { Stricture type } \\
\text { (patients) }\end{array}$ & $\begin{array}{l}\text { Management based on observed MRC } \\
\text { grading }\end{array}$ & No modified & $\begin{array}{l}\text { No modified } \\
\text { (theoretical) } \neq\end{array}$ \\
\hline I (4) & 4: endoscopy or surgery & 0 & 0 \\
II (7) & 7: endoscopy or combined procedures & 0 & 0 \\
III (7) & 4: percutaneous & 4 & 7 \\
& 2: endoscopy (MRC grade II) & & \\
IV (2) & 1: (MRC unsuccessful) & 1 & 2 \\
& 1: ? (MRC unsuccessful) & & \\
\hline
\end{tabular}

${ }^{\star}$ In this column are displayed the treatment choices which would have been made if MRC results had been taken into account;

†Number of treatment changes from actual management (see table 2).

$\ddagger$ Potential number of therapeutic changes if MRC had correctly graded all the patients.

undrained segments is poor. ${ }^{1}$ In this respect, pretreatment grading of hilar strictures, especially recognition of grade III and IV strictures, using a non-invasive imaging technique could select the best candidates for endoscopic and transhepatic palliation, as well as for conservative management.

Conventional ultrasonography can detect hilar malignancy in $80 \%$ of cases in expert hands. Spiral CT scan is even more accurate but cannot confidently determine operability. ${ }^{19}$ In addition, neither of these imaging procedures can give reliable information on stricture type. MRC is a non-invasive imaging technique that does not require administration of contrast medium and is based on the use of heavily T2 weighted sequences to depict static or slowly flowing fluid with high signal intensity whereas the background appears hypointense. Patient cooperation was demanded for breath-hold techniques in the earlier studies and some patients were not candidates for MRC because of this constraint. ${ }^{12}{ }^{13}$ Non-breath-hold techniques ${ }^{15}$ and rapid acquisition techniqueswithin a few seconds-have been developed. ${ }^{112021}$ The use of fast acquisition times almost completely eliminates motion artefacts. MRC can detect the presence of bile duct obstruction in $91-100 \%$ of cases and can determine the level of obstruction in $85-100 \%$ of cases. ${ }^{11}$ The MRC sequence used in this series was not up to date with the latest developments of the technique, for which our department was not equipped. We can speculate that slightly more complex sequences would have been able to improve the classification of some patients and eliminate non-diagnostic MRCs.

To our knowledge, this is the first prospective study to look at the use of MRC for pretreatment evaluation of malignant hilar obstruction and compare the results obtained with those for ERC. In our study, the magnetic resonance cholangiogram obtained for two patients was not of diagnostic quality $(10 \%)$; however, the technique correctly classified 14 of the remaining patients $(78 \%)$ with malignant hilar strictures and undergraded four $(22 \%)$. In all, $90 \%$ of the patients with type I and II strictures were correctly staged at MRC. As there was no stricture upgrading at MRC, had the pretreatment evaluation depended only on this technique, all 10 of these patients would have received the preferred treatment option-that is, endoscopic stenting. In the subgroup of patients with type III and IV strictures for whom endoscopic palliation is usually not recommended, MRC classified correctly four of the seven patients with a satisfactory MRC and underestimated extension in three (table 2). Had the management option (whether to place a stent endoscopically or not) depended in these three cases solely on MRC, the procedure would have been wrongly attempted in both type III patients undergraded as type II. In the patient with a type IV stricture undergraded as type III, endoscopic stenting would have been precluded, but transhepatic drainage would have been attempted with little chance of improvement. Table 3 summarises the most likely changes in management that MRC would have produced in this series. The hypothesis that MRC could influence patient management in a more appropriate way requires confirmation by prospective studies with management based on the results of MRC.

We conclude that MRC should be considered for planning treatment for malignant hilar strictures. By depicting high grade strictures for which endoscopic drainage is usually not indicated, it can preclude unnecessary and risky invasive imaging.

1 Levin B. Diagnosis and medical treatment of malignant disorders of the biliary tract. Semin Liver Dis 1987;7:328-33.

2 Imrie CW, Blumgart LH. Tumors of the extrahepatic biliary tree and pancreas. In: Millward-Sadler, Wright, Arthur, eds. Wright's liver and biliary disease. 3rd ed. London: WB Saunders, 1992:1516-39.

3 Barnerjee B, Teplick SK. Nonsurgical management of primary cholangiocarcinoma. Retrospective analysis of 40 primary cholangiocarcinoma. Ret.

4 Bismuth H, Castaing D, Traynor O. Resection or palliation: priority to surgery in the treatment of hilar cancer. World $\mathcal{F}$ Surg 1988;12:37-47.

5 Kozarek RA. Endoscopy in the management of malignant obstructive jaundice. Gastrointest Endosc Clin North Am 1996;6:153-76.

6 Reding R, Buard JL, Lebeau G, et al. Surgical management of 522 carcinomas of the extrahepatic bile ducts (gallbladder and periampullary tumors excluded). Ann Surg 1991;213:236-41.

7 Farley DR, Weaver AL, Nagorney DM. Natural history of unresected cholangiocarcinoma. Patient outcome after
noncurative intervention. Mayo Clin Proc 1995;70:425-9.

8 Chang WH, Kortan P, Haber GB. Outcome in patients with bifurcation tumors who undergo unilateral versus bilateral

hepatic duct drainage. Gastrointest Endosc 1998;47:354-62.

Bonnel D, Liguory C, Lefebvre JF, et al. Traitement percutané des sténoses

10 Ducreux M, Liguory C, Lefebvre JF, et al. Management of malignant hilar biliary obstruction by endoscopy. Results and pronostic factors. Dig Dis Sci 1992;37:778-83.

11 Reinhold C, Bret PM. Current status of MR cholangiography. AfR Am ₹ Roentgenol 1996;166:1285-95.

12 Hall-Crags MA, Allen CM, Owen CM, et al. MR cholangiography: clinical evaluation in 40 cases. Radiology 1993;189:423-7.

13 Ishizaki Y Wakayama T, Okada Y, et al. Magnetic resonance cholangiography for evaluation of obstructive jaundice. $\mathrm{Am}$ $\mathcal{f}$ Gastroenterol 1993;88:2072-7.

14 Guibaud L, Bret PM, Reinhold C, et al. Bile duct obstruction and choledocholithiasis: diagnosis whith MR cholangiography. Radiology 1995;197:109-15.

15 Macauley SE, Shulte SJ, Sekijama JH, et al. Evaluation of a non-breath-hold MR cholangiography. Radiology 1995; 196: $227-32$

16 Hadjis NS, Blenkharn JI, Alexander N, et al. Outcome of radical surgery in hilar cholangiocarcinoma. Surgery 1990; 107:597-604

17 Speer AG, Cotton PB, Russel RCG, et al. Randomised trial of endoscopic versus percutaneous stent insertion in malignant obstructive jaundice. Lancet 1987;ii:57-62.

18 Cotton PB. Endoscopic methods for relief of malignant obstructive jaundice. World $\mathcal{F}$ Surg 1984;8:854-61.

19 Gillams AR, Lees WR. Recent developments in biliary tract imaging. Gastrointest Endosc Clin North Am 1996;6:1-15.

20 Miyazaki T, Yamashita Y, Tsuchigame T, et al. MR cholangiopancreatography using HASTE (half Fourier acquisition single shot turbo-spin echo) sequences. AfR Am $\mathcal{F}$ Roentgenol 1996;166:1297-303.

21 Holzknecht N, Gauger J, Sackmann M, et al. Breath-hold MR cholangiography with snapshot techniques: prospective comparison with endoscopic retrograde cholangiography. Radiology 1998;206:657-64. 